\title{
STABILITY OF EQUILIBRIA OF A NON-LINEAR POPULATION DYNAMICAL MODEL
}

\author{
J.Z. FARKAS \\ Budapest University of Technology \\ Institute of Mathematics \\ Department of Differential Equations \\ Budapest, H-1521 \\ E-mail: farkas@math.bme.hu
}

\section{The characteristic equation}

Non-linear age-dependent biomathematical models have a recent history. Many biological phenomenons can be modelled better by non-linear differential equations. Age-specific mortality and fertility are among the most basic parameters of the theory of population dynamics and demography. In the present paper we shall consider the following McKendrick-type equation.

$$
\begin{gathered}
\frac{\partial p(t, a)}{\partial t}+\frac{\partial p(t, a)}{\partial a}=-\mu(a, P(t)) p(t, a) \\
p(0, a):=p_{0}(a),
\end{gathered}
$$

where $p(t, a)$ denotes the density of individuals of age $a$ at time $t$,

$$
P(t)=\int_{0}^{M} p(t, a) d a
$$

is the quantity of the total population at time $t ; \mu(a, P(t))$ denotes the intrinsic mortality and $\beta(a, P(t))$ the fertility, which depend on $P(t)$, in general, which makes the PDE (1.1) a non-linear one. (It is assumed that the vital rate functions $\beta, \mu$ are non-negative $C^{1}$ functions.)

The density of newborns at time $t$ is given by

$$
p(t, 0)=\int_{0}^{M} \beta(a, P(t)) p(t, a) d a .
$$


We assume a finite maximal age denoted by $M$.

Recall now the characteristic equation corresponding to the stationary solution of the system (1.1)-(1.3), $p_{1}(a)=p_{1}(0) e^{-\int_{0}^{a} \mu\left(s, P_{1}\right) d s}$ from ${ }^{1}$

$K(\lambda)=A_{11}(\lambda) A_{22}(\lambda)-A_{12}(\lambda) A_{21}(\lambda)+A_{12}(\lambda)+A_{21}(\lambda)=1$

where

$$
\begin{gathered}
A_{11}(\lambda)=\int_{0}^{M} e^{-\lambda a} e^{-\int_{0}^{a} \mu\left(s, P_{1}\right) d s} d a \\
A_{12}(\lambda)=-p_{1}(0) \int_{0}^{M} e^{-\lambda a} e^{-\int_{0}^{a} \mu\left(s, P_{1}\right) d s}\left(\int_{0}^{a} \mu_{P}^{\prime}\left(s, P_{1}\right) e^{\lambda s} d s\right) d a, \\
A_{21}(\lambda)=\int_{0}^{M} e^{-\lambda a} \beta\left(a, P_{1}\right) e^{-\int_{0}^{a} \mu\left(s, P_{1}\right) d s} d a \\
A_{22}(\lambda)=p_{1}(0)\left(\int_{0}^{M} \beta_{P}^{\prime}\left(a, P_{1}\right) e^{-\int_{0}^{a} \mu\left(s, P_{1}\right) d s} d a-\right. \\
\left.-\int_{0}^{M} e^{-\lambda a} \beta\left(a, P_{1}\right) e^{-\int_{0}^{a} \mu\left(s, P_{1}\right) d s} \int_{0}^{a} \mu_{P}^{\prime}\left(s, P_{1}\right) e^{\lambda s} d s d a\right) .
\end{gathered}
$$

Here $P_{1}=\int_{0}^{M} p_{1}(a) d a$ is the quantity of the total population.

Recall the following notations:

$$
\pi(a, P(t))=e^{-\int_{0}^{a} \mu(s, P(t)) d s}, R(P(t))=\int_{0}^{M} \beta(a, P(t)) \pi(a, P(t)) d a
$$

where the second term denotes the so called net reproductive number and $R\left(P_{1}\right)=1$ at any stationary solution, obviously.

\section{Conditions for stability}

Although Gurtin and McCamy deduced a characteristic equation in ${ }^{2}$ for a stationary solution of the system (1.1)-(1.3) in a completely different form, they didn't used it to proove stability except one very special example.

In the following two theorem we summarize our results obtained by the localization of the roots of this "new" characteristic equation (1.4), deduced by the author in ${ }^{1}$. 


\section{Theorem 2.1.}

In the case of $\mu(a, P)=m(a), \beta(a, P)$ general the stationary solution $p_{1}(a)$ at total population quantity $P_{1}$ is stable if $\beta^{\prime}\left(., P_{1}\right)<0$, if instead $\beta^{\prime}\left(., P_{1}\right)>0$ then it is unstable.

The following theorem gives a condition which implies instability of the stationary solution $p_{1}(a)$ for general $\mu(a, P), \beta(a, P)$.

\section{Theorem 2.2.}

If $R^{\prime}\left(P_{1}\right)>0$ holds then the stationary solution $p_{1}(a)$ at total population quantity $P_{1}$ is unstable.

\section{Acknowledgements}

The author is grateful to the Doctoral School of Applied Mathematics of the Budapest University of Technology which partially beared the costs of his participiation at the conference.

\section{References}

1. Farkas, M.: On the stability of stationary age distributions, Appl.Math. and Comput. 131 (2002), no.1 107-123

2. Gurtin, M.E.; MacCamy, R.C.: Non-linear age-dependent populations dynamics, Arch. Rat. Mech. Anal. 54 (1974), 281-300 